\title{
Does Racial Resentment Predict Anti-Black Discrimination?
}

\author{
L.J Zigerell \\ Assistant Professor \\ Illinois State University \\ 404 Schroeder Hall \\ Normal, IL 61790 \\ ljzigerell@ilstu.edu \\ @LJZigerell
}

\begin{abstract}
Racial resentment is a common social science measure for identifying the influence of racial attitudes on political phenomena. However, recent research has been mixed on whether racial resentment predicts racial discrimination in experiments. Data from three survey experiments from the archives of the Time-sharing Experiments for the Social Sciences indicated that the effect of racial resentment was not reliably higher in conditions with a black target than with a white target and that high levels of racial resentment did not reliably correlate with discrimination against black targets relative to white targets.
\end{abstract}

Keywords: racial resentment; symbolic racism; racism; race; discrimination

Acknowledgements. Data from the Time-sharing Experiments for the Social Sciences survey experiments described in this study were collected under National Science Foundation Grant 0818839, Jeremy Freese and Penny Visser, Principal Investigators. 


\section{Does Racial Resentment Predict Anti-Black Discrimination?}

Racial resentment is often used to assess whether racial attitudes influence political phenomena such as support for healthcare reform (Maxwell and Shields 2014), support for political rights for felons (Wilson et al. 2015), and support for gun control (Filindra and Kaplan 2016). Racial resentment is typically measured with agreement or disagreement with these statements:

1. Irish, Italians, Jewish and many other minorities overcame prejudice and worked their way up. Blacks should do the same without any special favors.

2. Generations of slavery and discrimination have created conditions that make it difficult for blacks to work their way out of the lower class.

3. Over the past few years, blacks have gotten less than they deserve.

4. It's really a matter of some people not trying hard enough; if blacks would only try harder they could be just as well off as whites.

An inferential concern about racial resentment research is that these items do not merely measure racial attitudes (see Sniderman and Tetlock 1986). For instance, a respondent who agreed that increased effort from blacks could eliminate the black/white gap could also believe that hard work could eliminate the gap between poor whites and wealthy whites. High racial resentment scores thus do not permit clear inferences about racial attitudes at the individual level.

However, it is not unreasonable to expect that, at the aggregate level, the set of persons with high racial resentment scores hold more antiblack attitudes than the set of persons with low racial resentment scores, because persons with antiblack bias should be disproportionately placed near or at the high end of the scale. Nonetheless, recent studies have produced conflicting inferences about racial resentment's ability to predict antiblack discrimination.

Meyer et al. (2016) reported on a survey that exploited a natural experiment in which two South Carolina Senate races were simultaneously held, with the Republican candidate being black male Tim Scott in one race and white male Lindsay Graham in the other race; racial resentment correlated similarly with support for both candidates in their respective races and did not correlate with preference for Scott or Graham in a hypothetical matchup of the two candidates against each other. Wallsten et al. (2017) reported on three survey experiments: the first experiment did not detect a difference in racial resentment's correlation with support for paying salaries to college athletes in the condition that displayed photos of black athletes, compared to the condition that displayed photos of white athletes; however, the subsequent experiments indicated that respondents at higher levels of racial resentment were more likely to oppose paying salaries to college athletes in the condition that presented a photo of one black athlete and two white athletes, compared to the condition that presented photos of three white athletes, and in the condition mentioning athletes with stereotypically black names, compared to the condition mentioning athletes with stereotypically white names. 
The present study continues this line of research by reporting on the three survey experiments available in the archives of the Time-sharing Experiments for the Social Sciences that included racial resentment items and permitted assessment of discrimination between black and white targets. ${ }^{1}$

\section{Overview of the Research Designs}

Reported results are for only white non-Hispanic respondents, with analyses weighted, and all model variables coded to run from 0 to 1 . Control conditions were omitted from the analysis because the focus is on how responses differ between exposure to black targets and white targets. Research designs assessed whether the effect of racial resentment is stronger in the black target condition than in the white target condition, and whether the set of respondents with high racial resentment scores discriminated against the black targets relative to the white targets. See the supplemental information file for item wording and other information about the surveys. The [redacted] Institutional Review Board has determined that the reported data analyses do not require IRB review because the analyses do not constitute human subjects research.

\section{Stephens (2011b)}

Stephens (2011b) is the best of the three studies for assessing the ability of racial resentment to predict racial discrimination, because that study had a relatively large sample, contained manipulation checks, and asked the racial resentment items in a pretest fielded one to sixteen days earlier than the racial discrimination experiment. See Stephens (2011a) for a different analysis of the survey experiment.

The experiment had a control condition, four white candidate conditions, and four black candidate conditions; the candidate was discussed in a news story that reflected one of four conditions: racially liberal, deracialized, implicitly racial, or explicitly racial. Reported outcome variables included vote likelihood for the target candidate and items measuring how well that candidate could be described as intelligent, inexperienced, trustworthy, hardworking, and fair; these adjective items were combined in a rating scale that had a Cronbach's alpha of 0.82 (Cronbach 1951).

Table 1 indicates that the coefficient for the interaction of racial resentment with candidate race did not reach statistical significance for vote likelihood or the rating scale, regardless of whether the sample was limited to respondents who passed the racial manipulation check and/or three other attention checks. ${ }^{2}$ Table 2 indicates that, within conditions, the

\footnotetext{
${ }^{1}$ The Banks (2010) study contained racial resentment items, but the manipulation in that experiment was of emotions and not the race of a target.

2 The three applied attention checks were whether the news article participants were shown concerned education, concerned the environment, and/or showed a candidate with glasses. The experiment contained an item a whether the candidate tried to appeal to racial feelings, but this item was not used to restrict the sample because responses to this item
} 
interaction term reached statistical significance only for vote likelihood in the implicitly racial condition; however, statistical tests did not detect discrimination against the black candidate among the set of persons at high levels of racial resentment in these models. Table 3 reports results for models with only the candidate race variable and condition controls, restricted to respondents at high values of racial resentment; no model detected a preference for the white candidate at or above the 50th, 75th, 90th, and 95th percentiles of racial resentment in the sample, but several models did detect a preference for the black candidate. To address the possibility that racial resentment is a better measure of racial attitudes among liberals (see Feldman and Huddy 2005), the bottom sections of Table 3 restrict the analysis to self-identified strong or not strong Democrats and self-identified liberals or extreme liberals; only one model detected discrimination against the black candidate, and this model had only six observations.

\section{[Tables 1, 2, and 3 about here]}

\section{Mcllwain and Caliendo (2010)}

The Mcllwain and Caliendo (2010) survey experiment had four conditions in which a black candidate competed against a target candidate who was either white and made no racial appeal, white and made an anti-black appeal, black and made no racial appeal, or black and made an appeal to racial authenticity. Reported analyses compared the white and black target candidates for only the nonracial appeal conditions because the anti-black appeal condition and authenticity appeal condition did not permit direct comparisons of the black candidate to the white candidate.

The survey included the eight items in the Symbolic Racism 2000 scale (Henry and Sears 2002); this scale contained the four items traditionally used for the racial resentment scale, and this four-item racial resentment scale was used in the analysis. The three reported outcome variables are: vote likelihood for the target candidate over the control candidate, preference for the target candidate over the control candidate based on responses to 0 -to100 feeling thermometers, and an indication of how much the target candidate rather than the control candidate reflects the respondent's ideal congressperson. See the study documentation and Mcllwain and Caliendo (2011) for more details on the experiment.

Results in Table 4 indicate that the interaction of racial resentment with the race of the target did not reach statistical significance for any outcome variable, and no model reported in Table 5 detected a preference for the white candidate over the black candidate in models restricted to persons at high levels of racial resentment and using only controls for the experimental conditions, for the full sample or for the sample restricted to selfidentified strong or not strong Democrats and self-identified liberals or extreme liberals.

[Tables 4 and 5 about here]

might influenced by the treatment, if, for instance, a black candidate is more likely than a white candidate to be perceived to try to appeal to racial feelings. 


\section{Baker (2011)}

The Baker (2011) survey experiment assessed racial discrimination in foreign aid and included two items modeled on the four traditional racial resentment items:

1. It's really a matter of some people not trying hard enough. If people in poor countries would only try harder, they could be just as well off as the United States.

2. Generations of colonialism and economic exploitation by rich countries have kept poor countries from becoming richer.

The experiment had a control condition, three conditions that discussed foreign aid going to poor African countries such as Cameroon and that displayed a photo of a black family, and three conditions that discussed foreign aid going to poor Eastern European countries such as Moldova and displayed a photo of a white family. One condition for each racial manipulation noted that American aid experts often instructed the poor how to use the aid, noted that American aid experts often instructed the poor how to use the aid and sometimes limited their choices, or provided no note about the American aid experts. Reported outcome variables indicate support for higher foreign aid spending in general, support for increasing foreign aid to the target country, level of agreement with the statement that the United States has a moral obligation to help foreign poor countries, and how much foreign aid to the target country is wasted. See Baker (2015) for more detail and more results regarding the experiment.

Tables 6 and 7 indicate that racial resentment in the Moldova condition correlated with less support for higher foreign aid spending in general, less support for increasing foreign aid to the target country, less agreement that the United States has a moral obligation to help foreign poor countries, and a higher estimate of how much of foreign aid to the target country is wasted; however, these correlations were not higher at $\mathrm{p}<0.05$ in the Cameroon condition, across all conditions (Table 6) or within conditions (Table 7). Moreover, Table 8 does not indicate discrimination against the black country among persons at the 50th, 75th, 90th, and 95th percentiles of racial resentment, for the full sample or for the sample restricted to self-identified strong or not strong Democrats and self-identified liberals or extreme liberals. ${ }^{3}$

[Tables 6 and 7 about here]

\section{Discussion}

Three survey experiments indicated that the effect of racial resentment in predicting support for a target did not reliably differ for black and white targets. Moreover, discrimination in favor of white targets over black targets was not detected among the set of persons at the 50th, 75th, 90th, and 95th percentiles of racial resentment, for both the full white samples and for white samples restricted to self-identified strong or not strong

3 The t-value was -2.19 for the 95th percentile for Democrats, but that model had only 9 observations, with a corresponding $\mathrm{p}$-value of $\mathrm{p}=0.059$. 
Democrats and self-identified liberals or extreme liberals. These results align more with Meyer et al. (2016) than Wallsten et al. (2017) in supporting skepticism about the ability of racial resentment to predict antiblack discrimination. 


\section{References}

Baker, Andy. 2012. Replication data for: "Racial Prejudice and Paternalism in Mass Support for Foreign Aid." http://www.tessexperiments.org/data/baker035.html

Baker, Andy. 2015. "Race, Paternalism, and Foreign Aid: Evidence from US Public Opinion." American Political Science Review 109(1): 93-109.

Banks, Antoine J. 2010. Replication data for: "The Public's Anger: White Racial Attitudes and Opinions toward Health Care Reform." http://www.tessexperiments.org/data/banks019.html.

Banks, Antoine J. 2014. "The Public's Anger: White Racial Attitudes and Opinions toward Health Care Reform." Political Behavior 36: 493-514.

Cronbach, Lee J. 1951. "Coefficient Alpha and the Internal Structure of Tests." Psychometrika 16(3): 297-334.

Feldman, Stanley, and Leonie Huddy. 2005. "Racial Resentment and White Opposition to Race-Conscious Programs: Principles or Prejudice?" American Journal of Political Science 49(1): 168-183.

Filindra, Alexandra, and Noah J. Kaplan. 2016. "Racial Resentment and Whites' Gun Policy Preferences in Contemporary America." Political Behavior 38(2): 255-275.

Maxwell, Angie, and Todd Shields. 2014. "The Fate of Obamacare: Racial Resentment, Ethnocentrism and Attitudes about Healthcare Reform." Race and Social Problems 6(4): 293-304.

McIlwain, Charlton, and Stephen Maynard Caliendo. 2010. Reproduction data for: "The Effects of Racist and Racial Appeals on White Voters." http://www.tessexperiments.org/data/mcilwain787.html.

Mcllwain, Charlton, and Stephen M. Caliendo. 2011. Race Appeal: How Candidates Invoke Race in US Political Campaigns. Philadelphia, PA: Temple University Press.

Meyer, Chase B., and J. David Woodard. 2016. "It's Not Race, It's Politics! A Natural Experiment Examining the Influence of Race in Electoral Politics." Social Science Quarterly. Early View.

Sniderman, Paul M., and Philip E. Tetlock. 1986. "Symbolic Racism: Problems of Motive Attribution in Political Analysis." Journal of Social Issues 42(2): 129-150.

Stephens, LaFleur. 2011a. "Beyond the Pale: White Americans' Conditional Response to the Norm of Racial Equality." Retrieved from: https://papers.ssrn.com/sol3/papers.cfm?abstract id=1944941. 
Stephens, LaFleur. 2011b. Reproduction data for: "Racial Appeals in a 'Post-Racial' Environment." http://www.tessexperiments.org/data/stephens072.html.

Wallsten, Kevin, Tatishe M. Nteta, Lauren A. McCarthy, and Melinda R. Tarsi. 2017. "Prejudice or Principled Conservatism? Racial Resentment and White Opinion toward Paying College Athletes." Political Research Quarterly. Early View.

Wilson, David C., Michael Leo Owens, and Darren W. Davis. 2015. "How Racial Attitudes and Ideology Affect Political Rights for Felons." Du Bois Review: Social Science Research on Race 12(1): 73-93. 
Table 1. Results from Stephens (2011b) across experimental conditions

\begin{tabular}{|c|c|c|c|c|c|c|}
\hline & \multicolumn{2}{|c|}{$\begin{array}{l}\text { No sample } \\
\text { restriction }\end{array}$} & \multicolumn{2}{|c|}{$\begin{array}{l}\text { Restricted to } \\
\text { respondents who } \\
\text { passed the racial } \\
\text { manipulation check }\end{array}$} & \multicolumn{2}{|c|}{$\begin{array}{l}\text { Restricted to } \\
\text { respondents who } \\
\text { passed the racial } \\
\text { manipulation check } \\
\text { and three attention } \\
\text { checks }\end{array}$} \\
\hline & $\begin{array}{c}\text { Vote } \\
\text { likelihood }\end{array}$ & $\begin{array}{l}\text { Rating } \\
\text { scale }\end{array}$ & $\begin{array}{c}\text { Vote } \\
\text { likelihood }\end{array}$ & $\begin{array}{l}\text { Rating } \\
\text { scale }\end{array}$ & $\begin{array}{c}\text { Vote } \\
\text { likelihood }\end{array}$ & $\begin{array}{l}\text { Rating } \\
\text { scale }\end{array}$ \\
\hline Black candidate & $\begin{array}{c}0.036 \\
(0.087)\end{array}$ & $\begin{array}{c}0.062 \\
(0.071)\end{array}$ & $\begin{array}{c}0.055 \\
(0.095)\end{array}$ & $\begin{array}{c}0.049 \\
(0.078)\end{array}$ & $\begin{array}{c}0.060 \\
(0.112)\end{array}$ & $\begin{array}{c}0.081 \\
(0.092)\end{array}$ \\
\hline $\begin{array}{l}\text { Racially liberal } \\
\text { condition }\end{array}$ & $\begin{array}{l}-0.330^{*} \\
(0.033)\end{array}$ & $\begin{array}{l}-0.277^{*} \\
(0.024)\end{array}$ & $\begin{array}{l}-0.351^{*} \\
(0.036)\end{array}$ & $\begin{array}{l}-0.282^{*} \\
(0.026)\end{array}$ & $\begin{array}{l}-0.372^{*} \\
(0.041)\end{array}$ & $\begin{array}{l}-0.270^{*} \\
(0.032)\end{array}$ \\
\hline Deracialized condition & $\begin{array}{l}-0.137^{*} \\
(0.031)\end{array}$ & $\begin{array}{l}-0.117^{*} \\
(0.025)\end{array}$ & $\begin{array}{l}-0.159 * \\
(0.036)\end{array}$ & $\begin{array}{l}-0.135^{*} \\
(0.029)\end{array}$ & $\begin{array}{l}-0.146^{*} \\
(0.040)\end{array}$ & $\begin{array}{l}-0.131^{*} \\
(0.032)\end{array}$ \\
\hline $\begin{array}{l}\text { Implicitly racial } \\
\text { condition }\end{array}$ & $\begin{array}{l}-0.063 \\
(0.032)\end{array}$ & $\begin{array}{l}-0.036 \\
(0.026)\end{array}$ & $\begin{array}{l}-0.070^{*} \\
(0.035)\end{array}$ & $\begin{array}{l}-0.061^{*} \\
(0.028)\end{array}$ & $\begin{array}{l}-0.068 \\
(0.041)\end{array}$ & $\begin{array}{l}-0.050 \\
(0.032)\end{array}$ \\
\hline Racial resentment & $\begin{array}{l}-0.102 \\
(0.102)\end{array}$ & $\begin{array}{l}-0.037 \\
(0.083)\end{array}$ & $\begin{array}{l}-0.104 \\
(0.104)\end{array}$ & $\begin{array}{l}-0.044 \\
(0.084)\end{array}$ & $\begin{array}{l}-0.088 \\
(0.126)\end{array}$ & $\begin{array}{l}-0.023 \\
(0.100)\end{array}$ \\
\hline $\begin{array}{l}\text { Black candidate X } \\
\text { Racial resentment }\end{array}$ & $\begin{array}{c}0.024 \\
(0.132)\end{array}$ & $\begin{array}{c}0.019 \\
(0.107)\end{array}$ & $\begin{array}{l}-0.007 \\
(0.143)\end{array}$ & $\begin{array}{c}0.065 \\
(0.117)\end{array}$ & $\begin{array}{l}-0.038 \\
(0.169)\end{array}$ & $\begin{array}{l}-0.001 \\
(0.141)\end{array}$ \\
\hline Constant & $\begin{array}{l}0.571^{*} \\
(0.069)\end{array}$ & $\begin{array}{l}0.540^{*} \\
(0.057)\end{array}$ & $\begin{array}{l}0.587^{*} \\
(0.071)\end{array}$ & $\begin{array}{l}0.558^{*} \\
(0.057)\end{array}$ & $\begin{array}{l}.593^{*} \\
(0.083)\end{array}$ & $\begin{array}{l}0.558^{*} \\
(0.066)\end{array}$ \\
\hline $\mathrm{N}$ & 791 & 776 & 662 & 650 & 488 & 478 \\
\hline
\end{tabular}

Note: Cells indicate coefficients and standard errors from linear regressions. All variables range from 0 to 1 . The analysis omitted the control condition, and the omitted category for the remaining conditions is the explicitly racial condition. Bold and asterisks indicate $\mathrm{p}<0.05$ with a two-tailed test. The sample was restricted to non-Hispanic whites. 
Table 2. Results from Stephens (2011b) within experimental conditions

\begin{tabular}{|c|c|c|c|c|c|c|}
\hline & \multicolumn{2}{|c|}{ No sample restriction } & \multicolumn{2}{|c|}{$\begin{array}{l}\text { Restricted to } \\
\text { respondents who } \\
\text { passed the racial } \\
\text { manipulation check }\end{array}$} & \multicolumn{2}{|c|}{$\begin{array}{l}\text { Restricted to } \\
\text { respondents who passed } \\
\text { the racial manipulation } \\
\text { check and three } \\
\text { attention checks }\end{array}$} \\
\hline & $\begin{array}{c}\text { Vote } \\
\text { likelihood }\end{array}$ & $\begin{array}{l}\text { Rating } \\
\text { scale }\end{array}$ & $\begin{array}{c}\text { Vote } \\
\text { likelihood }\end{array}$ & $\begin{array}{l}\text { Rating } \\
\text { scale }\end{array}$ & $\begin{array}{c}\text { Vote } \\
\text { likelihood }\end{array}$ & $\begin{array}{l}\text { Rating } \\
\text { scale }\end{array}$ \\
\hline \multicolumn{7}{|c|}{ Racially liberal condition } \\
\hline Black candidate & $\begin{array}{c}-0.282 \\
(0.199) \\
\end{array}$ & $\begin{array}{l}-0.156 \\
(0.121) \\
\end{array}$ & $\begin{array}{c}-0.216 \\
90.224) \\
\end{array}$ & $\begin{array}{l}-0.116 \\
(0.126)\end{array}$ & $\begin{array}{l}-0.253 \\
(0.248)\end{array}$ & $\begin{array}{c}-0.225 \\
(0.149) \\
\end{array}$ \\
\hline Racial resentment & $\begin{array}{l}-0.484^{*} \\
(0.201)\end{array}$ & $\begin{array}{l}-0.487^{*} \\
(0.132)\end{array}$ & $\begin{array}{l}-0.483^{*} \\
(0.204) \\
\end{array}$ & $\begin{array}{l}-0.489 * \\
(0.132)\end{array}$ & $\begin{array}{l}-0.579 * \\
(0.266)\end{array}$ & $\begin{array}{l}-0.617^{*} \\
(0.150)\end{array}$ \\
\hline $\begin{array}{l}\text { Black candidate X Racial } \\
\text { resentment }\end{array}$ & $\begin{array}{c}0.428 \\
(0.301) \\
\end{array}$ & $\begin{array}{c}0.296 \\
(0.173) \\
\end{array}$ & $\begin{array}{c}0.309 \\
(0.340) \\
\end{array}$ & $\begin{array}{c}0.270 \\
(0.186) \\
\end{array}$ & $\begin{array}{c}0.318 \\
(0.352) \\
\end{array}$ & $\begin{array}{c}0.434 \\
(0.232) \\
\end{array}$ \\
\hline Constant & $\begin{array}{l}0.517^{*} \\
(0.142) \\
\end{array}$ & $\begin{array}{l}0.572^{*} \\
(0.094)\end{array}$ & $\begin{array}{c}0.509 \\
(0.144) \\
\end{array}$ & $\begin{array}{l}0.574 * \\
(0.095) \\
\end{array}$ & $\begin{array}{l}0.571^{*} \\
(0.194) \\
\end{array}$ & $\begin{array}{l}0.677^{*} \\
(0.108) \\
\end{array}$ \\
\hline $\mathrm{N}$ & 198 & 196 & 163 & 162 & 106 & 105 \\
\hline \multicolumn{7}{|c|}{ Deracialized condition } \\
\hline Black candidate & $\begin{array}{c}-0.145 \\
(0.151) \\
\end{array}$ & $\begin{array}{c}0.040 \\
(0.121) \\
\end{array}$ & $\begin{array}{l}-0.136 \\
(0.195) \\
\end{array}$ & $\begin{array}{l}-0.116 \\
(0.126) \\
\end{array}$ & $\begin{array}{l}-0.130 \\
(0.245) \\
\end{array}$ & $\begin{array}{c}0.170 \\
(0.164) \\
\end{array}$ \\
\hline Racial resentment & $\begin{array}{c}-0.284 \\
(0.164) \\
\end{array}$ & $\begin{array}{c}0.003 \\
(0.149) \\
\end{array}$ & $\begin{array}{l}-0.284 \\
(0.164) \\
\end{array}$ & $\begin{array}{l}-0.489 * \\
(0.132) \\
\end{array}$ & $\begin{array}{l}-0.300 \\
(0.191)\end{array}$ & $\begin{array}{c}0.057 \\
(0.156) \\
\end{array}$ \\
\hline $\begin{array}{l}\text { Black candidate X Racial } \\
\text { resentment }\end{array}$ & $\begin{array}{c}0.238 \\
(0.229) \\
\end{array}$ & $\begin{array}{l}-0.039 \\
(0.197)\end{array}$ & $\begin{array}{c}0.163 \\
(0.280) \\
\end{array}$ & $\begin{array}{c}0.270 \\
(0.186)\end{array}$ & $\begin{array}{c}0.174 \\
(0.358)\end{array}$ & $\begin{array}{c}-0.241 \\
(0.257) \\
\end{array}$ \\
\hline Constant & $\begin{array}{l}0.572^{*} \\
(0.104) \\
\end{array}$ & $\begin{array}{l}.424 * \\
(0.086) \\
\end{array}$ & $\begin{array}{l}0.572^{*} \\
(0.104) \\
\end{array}$ & $\begin{array}{l}0.574 * \\
(0.094) \\
\end{array}$ & $\begin{array}{l}0.598^{*} \\
(0.111) \\
\end{array}$ & $\begin{array}{l}0.399 * \\
(0.088) \\
\end{array}$ \\
\hline $\mathrm{N}$ & 203 & 197 & 167 & 163 & 135 & 132 \\
\hline \multicolumn{7}{|c|}{ Implicitly racial condition } \\
\hline Black candidate & $\begin{array}{l}0.441 * \\
(0.140)\end{array}$ & $\begin{array}{c}0.292^{*} \\
(0.141)\end{array}$ & $\begin{array}{l}0.500^{*} \\
(0.151)\end{array}$ & $\begin{array}{l}0^{3} 373^{*} \\
(0.151)\end{array}$ & $\begin{array}{l}0.528^{*} \\
(0.174)\end{array}$ & $\begin{array}{l}0.436^{*} \\
(0.176)\end{array}$ \\
\hline Racial resentment & $\begin{array}{l}0.449^{*} \\
(0.146)\end{array}$ & $\begin{array}{l}0.443^{*} \\
(0.132)\end{array}$ & $\begin{array}{l}0.456^{*} \\
(0.155)\end{array}$ & $\begin{array}{l}\text { 0.461* } \\
(0.140)\end{array}$ & $\begin{array}{l}0.5028^{*} \\
(0.182)\end{array}$ & $\begin{array}{l}0.517^{*} \\
(0.158)\end{array}$ \\
\hline $\begin{array}{l}\text { Black candidate X Racial } \\
\text { resentment }\end{array}$ & $\begin{array}{c}-0.432 \\
(0.221) \\
\end{array}$ & $\begin{array}{l}-0.148 \\
(0.216)\end{array}$ & $\begin{array}{l}-0.504^{*} \\
(0.237) \\
\end{array}$ & $\begin{array}{l}-0.266 \\
(0.228) \\
\end{array}$ & $\begin{array}{l}-0.602^{*} \\
(0.276) \\
\end{array}$ & $\begin{array}{c}-0.412 \\
(0.264) \\
\end{array}$ \\
\hline Constant & $\begin{array}{c}0.084 \\
(0.095) \\
\end{array}$ & $\begin{array}{c}0.114 \\
(0.091)\end{array}$ & $\begin{array}{c}0.082 \\
(0.099) \\
\end{array}$ & $\begin{array}{c}0.101 \\
(0.095) \\
\end{array}$ & $\begin{array}{c}0.083 \\
(0.113) \\
\end{array}$ & $\begin{array}{c}0.103 \\
(0.110) \\
\end{array}$ \\
\hline $\mathrm{N}$ & 200 & 195 & 178 & 173 & 123 & 119 \\
\hline \multicolumn{7}{|c|}{ Explicitly racial condition } \\
\hline Black candidate & $\begin{array}{c}0.196 \\
(0.148) \\
\end{array}$ & $\begin{array}{c}0.151 \\
(0.111) \\
\end{array}$ & $\begin{array}{c}0.163 \\
(0.152) \\
\end{array}$ & $\begin{array}{c}0.064 \\
(0.113) \\
\end{array}$ & $\begin{array}{c}0.169 \\
(0.170)\end{array}$ & $\begin{array}{c}0.060 \\
(0.123) \\
\end{array}$ \\
\hline Racial resentment & $\begin{array}{c}0.025 \\
(0.195)\end{array}$ & $\begin{array}{c}-0.19 \\
(0.134)\end{array}$ & $\begin{array}{c}0.031 \\
(0.200)\end{array}$ & $\begin{array}{l}-0.050 \\
(0.132)\end{array}$ & $\begin{array}{c}0.081 \\
(0.230) \\
\end{array}$ & $\begin{array}{l}-0.028 \\
(0.150)\end{array}$ \\
\hline $\begin{array}{l}\text { Black candidate X Racial } \\
\text { resentment }\end{array}$ & $\begin{array}{l}-0.227 \\
(0.238)\end{array}$ & $\begin{array}{l}-0.142 \\
(0.174)\end{array}$ & $\begin{array}{l}-0.162 \\
(0.250) \\
\end{array}$ & $\begin{array}{c}0.049 \\
(0.173)\end{array}$ & $\begin{array}{l}0.159 \\
(0.288) \\
\end{array}$ & $\begin{array}{c}0.037 \\
(0.190) \\
\end{array}$ \\
\hline Constant & $\begin{array}{l}0.486^{*} \\
(0.121)\end{array}$ & $\begin{array}{l}0.532^{*} \\
(0.084)\end{array}$ & $\begin{array}{l}0.494^{*} \\
(0.125)\end{array}$ & $\begin{array}{l}0.559 * \\
(0.082)\end{array}$ & $\begin{array}{l}0.472^{*} \\
(0.139)\end{array}$ & $\begin{array}{l}0.560^{*} \\
(0.091)\end{array}$ \\
\hline $\mathrm{N}$ & 190 & 188 & 154 & 152 & 124 & 122 \\
\hline
\end{tabular}

Note: Cells indicate coefficients and standard errors from linear regressions. All variables range from 0 to 1 . The analysis omitted the control condition, and the omitted category for the remaining conditions is the explicitly racial condition. Bold and asterisks indicate $\mathrm{p}<0.05$ with a two-tailed test. The sample was restricted to non-Hispanic whites. 
Table 3: Results for the black target candidate variable from Stephens (2011b) for high levels of racial resentment

\begin{tabular}{|c|c|c|c|c|c|c|}
\hline & \multicolumn{2}{|c|}{$\begin{array}{l}\text { No sample } \\
\text { restriction }\end{array}$} & \multicolumn{2}{|c|}{$\begin{array}{l}\text { Restricted to } \\
\text { respondents who } \\
\text { passed the racial } \\
\text { manipulation check }\end{array}$} & \multicolumn{2}{|c|}{$\begin{array}{c}\text { Restricted to } \\
\text { respondents who } \\
\text { passed the racial } \\
\text { manipulation check } \\
\text { and three attention } \\
\text { checks }\end{array}$} \\
\hline & $\begin{array}{c}\text { Vote } \\
\text { likelihood }\end{array}$ & $\begin{array}{l}\text { Rating } \\
\text { scale }\end{array}$ & $\begin{array}{c}\text { Vote } \\
\text { likelihood }\end{array}$ & $\begin{array}{l}\text { Rating } \\
\text { scale }\end{array}$ & $\begin{array}{c}\text { Vote } \\
\text { likelihood }\end{array}$ & $\begin{array}{c}\text { Rating } \\
\text { scale }\end{array}$ \\
\hline \multicolumn{7}{|c|}{ Full sample of whites } \\
\hline $\begin{array}{l}\text { Top } 50 \% \text { on the racial } \\
\text { resentment scale }\end{array}$ & $\begin{array}{c}0.051 \\
(0.029) \\
\end{array}$ & $\begin{array}{l}0.076 * \\
(0.022) \\
\end{array}$ & $\begin{array}{c}0.057 \\
(0.038) \\
\end{array}$ & $\begin{array}{c}0.117^{*} \\
(0.028) \\
\end{array}$ & $\begin{array}{c}0.042 \\
(0.043) \\
\end{array}$ & $\begin{array}{l}0.096^{*} \\
(0.034) \\
\end{array}$ \\
\hline $\begin{array}{l}\text { Top } 25 \% \text { on the racial } \\
\text { resentment scale }\end{array}$ & $\begin{array}{c}0.037 \\
(0.039)\end{array}$ & $\begin{array}{l}0.088^{*} \\
(0.031)\end{array}$ & $\begin{array}{c}0.031 \\
(0.042)\end{array}$ & $\begin{array}{l}0.119 * \\
(0.033)\end{array}$ & $\begin{array}{c}0.012 \\
(0.051)\end{array}$ & $\begin{array}{c}0.097 \\
(0.041)\end{array}$ \\
\hline $\begin{array}{l}\text { Top } 10 \% \text { on the racial } \\
\text { resentment scale }\end{array}$ & $\begin{array}{c}0.021 \\
(0.086) \\
\end{array}$ & $\begin{array}{c}0.017 \\
(0.062)\end{array}$ & $\begin{array}{c}0.018 \\
(0.095) \\
\end{array}$ & $\begin{array}{c}0.039 \\
(0.069) \\
\end{array}$ & $\begin{array}{l}-0.048 \\
(0.111) \\
\end{array}$ & $\begin{array}{l}-0.043 \\
(0.090) \\
\end{array}$ \\
\hline $\begin{array}{l}\text { Top } 5 \% \text { on the racial } \\
\text { resentment scale }\end{array}$ & $\begin{array}{l}-0.074 \\
(0.096) \\
\end{array}$ & $\begin{array}{c}-0.007 \\
(0.090) \\
\end{array}$ & $\begin{array}{l}-0.083 \\
(0.105) \\
\end{array}$ & $\begin{array}{l}-0.007 \\
(0.098) \\
\end{array}$ & $\begin{array}{l}-0.117 \\
(0.143) \\
\end{array}$ & $\begin{array}{l}-0.113 \\
(0.123) \\
\end{array}$ \\
\hline \multicolumn{7}{|c|}{ White sample restricted to self-identified strong Democrats and not strong Democrats } \\
\hline $\begin{array}{l}\text { Top } 50 \% \text { on the racial } \\
\text { resentment scale }\end{array}$ & $\begin{array}{c}0.092 \\
(0.062) \\
\end{array}$ & $\begin{array}{c}0.125 * \\
(0.044) \\
\end{array}$ & $\begin{array}{c}0.131 \\
(0.069) \\
\end{array}$ & $\begin{array}{c}0.143^{*} \\
(0.050)\end{array}$ & $\begin{array}{c}0.027 \\
(0.067) \\
\end{array}$ & $\begin{array}{l}0.108^{*} \\
(0.054)\end{array}$ \\
\hline $\begin{array}{l}\text { Top } 25 \% \text { on the racial } \\
\text { resentment scale }\end{array}$ & $\begin{array}{l}0.203^{*} \\
(0.075) \\
\end{array}$ & $\begin{array}{l}0.213^{*} \\
(0.056) \\
\end{array}$ & $\begin{array}{l}0.218 * \\
(0.089) \\
\end{array}$ & $\begin{array}{l}0.235^{*} \\
(0.059)\end{array}$ & $\begin{array}{c}0.166 \\
(0.097) \\
\end{array}$ & $\begin{array}{l}0.218^{*} \\
(0.065)\end{array}$ \\
\hline $\begin{array}{l}\text { Top } 10 \% \text { on the racial } \\
\text { resentment scale }\end{array}$ & $\begin{array}{c}0.176 \\
(0.115)\end{array}$ & $\begin{array}{c}0.100 \\
(0.102)\end{array}$ & $\begin{array}{c}0.204 \\
(0.126)\end{array}$ & $\begin{array}{c}0.173 \\
(0.112) \\
\end{array}$ & $\begin{array}{c}0.277 \\
(0.134)\end{array}$ & $\begin{array}{c}0.229 \\
(0.122)\end{array}$ \\
\hline $\begin{array}{l}\text { Top } 5 \% \text { on the racial } \\
\text { resentment scale }\end{array}$ & $\begin{array}{c}0.120 \\
(0.147) \\
\end{array}$ & $\begin{array}{c}-0.002 \\
(0.145) \\
\end{array}$ & $\begin{array}{c}0.275 \\
(0.201) \\
\end{array}$ & $\begin{array}{c}0.158 \\
(0.227) \\
\end{array}$ & $\begin{array}{c}0.502^{*} \\
(0.189) \\
\end{array}$ & $\begin{array}{c}0.374 \\
(0.264) \\
\end{array}$ \\
\hline \multicolumn{7}{|c|}{ White sample restricted to persons who self-identified as liberal or extremely liberal } \\
\hline $\begin{array}{l}\text { Top } 50 \% \text { on the racial } \\
\text { resentment scale }\end{array}$ & $\begin{array}{c}0.023 \\
(0.077)\end{array}$ & $\begin{array}{c}0.080 \\
(0.055)\end{array}$ & $\begin{array}{c}0.092 \\
(0.090)\end{array}$ & $\begin{array}{c}0.105 \\
(0.073)\end{array}$ & $\begin{array}{c}0.125 \\
(0.121)\end{array}$ & $\begin{array}{l}0.135^{*} \\
(0.063)\end{array}$ \\
\hline $\begin{array}{l}\text { Top } 25 \% \text { on the racial } \\
\text { resentment scale }\end{array}$ & $\begin{array}{c}-0.001 \\
(0.100) \\
\end{array}$ & $\begin{array}{c}0.113 \\
(0.071) \\
\end{array}$ & $\begin{array}{c}0.138 \\
(0.115) \\
\end{array}$ & $\begin{array}{c}0.219 * \\
(0.077) \\
\end{array}$ & $\begin{array}{c}-0.075 \\
(0.168) \\
\end{array}$ & $\begin{array}{r}0.208^{*} \\
(0.065)\end{array}$ \\
\hline $\begin{array}{l}\text { Top } 10 \% \text { on the racial } \\
\text { resentment scale }\end{array}$ & $\begin{array}{c}0.116 \\
(0.153) \\
\end{array}$ & $\begin{array}{c}0.168 \\
(0.085) \\
\end{array}$ & $\begin{array}{c}0.165 \\
(0.298) \\
\end{array}$ & $\begin{array}{c}0.236 \\
(0.122) \\
\end{array}$ & $\begin{array}{l}-0.331 \\
(0.133) \\
\end{array}$ & $\begin{array}{c}0.096 \\
(0.074) \\
\end{array}$ \\
\hline $\begin{array}{l}\text { Top 5\% on the racial } \\
\text { resentment scale }\end{array}$ & $\begin{array}{c}-0.151 \\
(0.180)\end{array}$ & $\begin{array}{l}-0.261^{*} \\
(0.065)\end{array}$ & $\begin{array}{l}-0.500 \\
(\mathrm{~N} / \mathrm{A})\end{array}$ & $\begin{array}{l}-0.289 \\
(\mathrm{~N} / \mathrm{A})\end{array}$ & $\begin{array}{l}-0.000 \\
(\mathrm{~N} / \mathrm{A})\end{array}$ & $\begin{array}{l}0.300 \\
(\mathrm{~N} / \mathrm{A})\end{array}$ \\
\hline
\end{tabular}

Note: Cells indicate coefficients and standard errors for the candidate race coefficient from linear regressions that included controls for experimental conditions. All variables range from 0 to 1 . Bold and asterisks indicate $\mathrm{p}<0.05$ with a two-tailed test. The sample was restricted to non-Hispanic whites. Percentiles for subgroups are based on the distribution of racial resentment within that subgroup. N/A indicates that the statistical software did not report standard errors. 
Table 4. Results for McIlwain and Caliendo (2010)

\begin{tabular}{|l|c|c|c|}
\hline & Vote likelihood & $\begin{array}{c}\text { Feeling } \\
\text { thermometer } \\
\text { difference }\end{array}$ & $\begin{array}{c}\text { How well the } \\
\text { candidate } \\
\text { represents the } \\
\text { ideal } \\
\text { congressperson }\end{array}$ \\
\hline \multicolumn{2}{|c|}{ Four-item racial resentment scale } \\
\hline Black target candidate & 1.856 & 0.150 & 0.116 \\
Racial resentment & $(1.276)$ & $(0.093)$ & $(0.113)$ \\
\hline Black target candidate X Racial & 2.166 & 0.153 & -0.006 \\
resentment & $(1.463)$ & $(0.118)$ & $(0.103)$ \\
\hline Constant & -2.427 & -0.247 & -0.114 \\
& $(2.112)$ & $0.172)$ & $(0.200)$ \\
\hline $\mathrm{N}$ & -1.279 & $\mathbf{0 . 5 0 9 *}$ & $\mathbf{0 . 4 8 2}$ \\
& $(0.868)$ & $\mathbf{( 0 . 0 6 2 )}$ & $\mathbf{( 0 . 0 4 8 )}$ \\
\hline
\end{tabular}

Note: Cells indicate coefficients and standard errors for a logistic regression (model 1) and linear regressions (models 2 and 3 ). All variables range from 0 to 1 . Bold and asterisks indicate $\mathrm{p}<0.05$ with a two-tailed test. The sample was restricted to non-Hispanic whites. 
Table 5: Results for the black target candidate variable from Mcllwain and Caliendo (2010) for high levels of racial resentment

\begin{tabular}{|c|c|c|c|}
\hline & \multicolumn{3}{|c|}{ Four-item racial resentment } \\
\hline & $\begin{array}{c}\text { Vote } \\
\text { likelihood }\end{array}$ & $\begin{array}{c}\text { Feeling } \\
\text { thermometer } \\
\text { difference }\end{array}$ & $\begin{array}{l}\text { How well the } \\
\text { candidate } \\
\text { represents the } \\
\text { ideal } \\
\text { congressperson }\end{array}$ \\
\hline \multicolumn{4}{|c|}{ Full sample of whites } \\
\hline Top $50 \%$ on the racial resentment scale & $\begin{array}{c}0.261 \\
(0.592)\end{array}$ & $\begin{array}{c}-0.002 \\
(0.061)\end{array}$ & $\begin{array}{c}0.074 \\
(0.071)\end{array}$ \\
\hline Top $25 \%$ on the racial resentment scale & $\begin{array}{c}0.084 \\
(0.684)\end{array}$ & $\begin{array}{l}-0.033 \\
(0.068)\end{array}$ & $\begin{array}{c}-0.022 \\
(0.071)\end{array}$ \\
\hline Top $10 \%$ on the racial resentment scale & $\begin{array}{c}-0.762 \\
(1.334)\end{array}$ & $\begin{array}{c}-0.188 \\
(0.130) \\
\end{array}$ & $\begin{array}{c}-0.077 \\
(0.125) \\
\end{array}$ \\
\hline Top 5\% on the racial resentment scale & \multicolumn{3}{|c|}{ Same results as for the top $10 \%$} \\
\hline \multicolumn{4}{|c|}{ White sample restricted to self-identified strong Democrats and not strong Democrats } \\
\hline Top $50 \%$ on the racial resentment scale & $\begin{array}{c}0.036 \\
(1.302)\end{array}$ & $\begin{array}{c}0.035 \\
(0.066) \\
\end{array}$ & $\begin{array}{c}0.192 \\
(0.212) \\
\end{array}$ \\
\hline Top $25 \%$ on the racial resentment scale & $\begin{array}{c}3.041 \\
(1.596) \\
\end{array}$ & $\begin{array}{c}0.215 \\
(0.058) \\
\end{array}$ & $\begin{array}{c}0.208 \\
(0.412) \\
\end{array}$ \\
\hline Top $10 \%$ on the racial resentment scale & --- & $\begin{array}{c}0.285 \\
(0.041)\end{array}$ & $\begin{array}{c}0.145 \\
(0.437)\end{array}$ \\
\hline Top 5\% on the racial resentment scale & --- & --- & $\begin{array}{r}-0.353 \\
(\mathrm{~N} / \mathrm{A}) \\
\end{array}$ \\
\hline \multicolumn{4}{|c|}{ White sample restricted to persons who self-identified as liberal or extremely liberal } \\
\hline Top $50 \%$ on the racial resentment scale & $\begin{array}{c}-0.312 \\
(1.457) \\
\end{array}$ & $\begin{array}{c}-0.014 \\
(0.112) \\
\end{array}$ & $\begin{array}{c}0.616 \\
(0.299) \\
\end{array}$ \\
\hline Top $25 \%$ on the racial resentment scale & --- & $\begin{array}{c}0.351 \\
(0.182)\end{array}$ & $\begin{array}{c}1.101 \\
(0.128)\end{array}$ \\
\hline Top $10 \%$ on the racial resentment scale & --- & $\begin{array}{c}0.432 \\
(0.172)\end{array}$ & $\begin{array}{c}1.090 \\
(0.138)\end{array}$ \\
\hline Top $5 \%$ on the racial resentment scale & --- & --- & --- \\
\hline
\end{tabular}

Note: Cells indicate coefficients and standard errors for the candidate race coefficient from logistic (model 1) and linear (models 2 and 3) regressions that included only the candidate race variable as a predictor. All variables range from 0 to 1 . Bold and asterisks indicate $\mathrm{p}<0.05$ with a two-tailed test. The sample was restricted to non-Hispanic whites. N/A indicates that the statistical software did not report standard errors, and dashes indicate that the software did not report any results for that model. Percentiles for subgroups are based on the distribution of racial resentment within that subgroup. 
Table 6. Results from Baker (2011) across experimental conditions

\begin{tabular}{|l|c|c|c|c|}
\hline & $\begin{array}{c}\text { Support for } \\
\text { higher foreign } \\
\text { aid spending } \\
\text { in general }\end{array}$ & $\begin{array}{c}\text { Support for } \\
\text { increasing } \\
\text { foreign aid to } \\
\text { the target } \\
\text { country }\end{array}$ & $\begin{array}{c}\text { Moral } \\
\text { obligation to } \\
\text { help foreign } \\
\text { poor } \\
\text { countries }\end{array}$ & $\begin{array}{c}\text { Foreign aid to } \\
\text { the target } \\
\text { country is not } \\
\text { wasted }\end{array}$ \\
\hline Cameroon & 0.010 & 0.044 & 0.051 & 0.021 \\
$(0.031)$ & $(0.028)$ & $(0.034)$ & $(0.030)$ \\
\hline No paternalism & 0.002 & 0.004 & $0.040^{*}$ & -0.031 \\
condition & $(0.017)$ & $(0.015)$ & $(0.018)$ & $(0.016)$ \\
\hline Limiting paternalism & -0.018 & -0.016 & -0.003 & 0.004 \\
condition & $(0.017)$ & $(0.015)$ & $(0.019)$ & $0.017)$ \\
\hline Racial resentment & $\mathbf{- 0 . 5 8 9}$ & $\mathbf{- 0 . 5 3 8}$ & $\mathbf{- 0 . 7 0 7}$ & $\mathbf{- 0 . 3 4 5}$ \\
\hline Cameroon X Racial & $(\mathbf{0 . 0 4 6 )}$ & $\mathbf{( 0 . 0 4 0 )}$ & $\mathbf{( 0 . 0 4 9 )}$ & $\mathbf{( 0 . 0 4 6 )}$ \\
resentment & 0.035 & -0.015 & -0.011 & -0.047 \\
\hline Constant & $(0.061)$ & $(0.056)$ & $(0.071)$ & $(0.061)$ \\
\hline $\mathrm{N}$ & $\mathbf{0 . 6 1 0 *}$ & $\mathbf{0 . 6 0 0} *$ & $\mathbf{0 . 7 3 2} *$ & $\mathbf{0 . 6 3 0}$ \\
\hline
\end{tabular}

Note: Cells indicate coefficients and standard errors from linear regressions. All variables range from 0 to 1 . The analysis omitted the control condition, and the omitted category for the remaining conditions is the instructive paternalism only condition. Bold and asterisks indicate $\mathrm{p}<0.05$ with a two-tailed test. The sample was restricted to non-Hispanic whites. 
Table 7. Results from Baker (2011) within experimental conditions

\begin{tabular}{|c|c|c|c|c|}
\hline & $\begin{array}{l}\text { Support for } \\
\text { higher foreign } \\
\text { aid spending } \\
\text { in general }\end{array}$ & $\begin{array}{l}\text { Support for } \\
\text { increasing } \\
\text { foreign aid to } \\
\text { the target } \\
\text { country }\end{array}$ & $\begin{array}{c}\text { Moral } \\
\text { obligation to } \\
\text { help foreign } \\
\text { poor } \\
\text { countries }\end{array}$ & $\begin{array}{c}\text { Foreign aid to } \\
\text { the target } \\
\text { country is not } \\
\text { wasted }\end{array}$ \\
\hline \multicolumn{5}{|c|}{ No paternalism condition } \\
\hline Cameroon & $\begin{array}{l}-0.026 \\
(0.048)\end{array}$ & $\begin{array}{c}0.007 \\
(0.045)\end{array}$ & $\begin{array}{c}0.001 \\
(0.051)\end{array}$ & $\begin{array}{l}-0.012 \\
(0.048)\end{array}$ \\
\hline Racial resentment & $\begin{array}{l}-0.627^{*} \\
(0.060)\end{array}$ & $\begin{array}{l}-0.586^{*} \\
(0.053)\end{array}$ & $\begin{array}{l}-0.794^{*} \\
(0.057)\end{array}$ & $\begin{array}{l}-0.312^{*} \\
(0.064)\end{array}$ \\
\hline $\begin{array}{l}\text { Cameroon X Racial } \\
\text { resentment }\end{array}$ & $\begin{array}{c}0.119 \\
(0.094)\end{array}$ & $\begin{array}{c}0.105 \\
(0.088)\end{array}$ & $\begin{array}{c}0.155 \\
(0.114)\end{array}$ & $\begin{array}{c}0.007 \\
(0.099)\end{array}$ \\
\hline Constant & $\begin{array}{l}0.628^{*} \\
(0.033)\end{array}$ & $\begin{array}{l}0.617^{*} \\
(0.029)\end{array}$ & $\begin{array}{l}0.799^{*} \\
(0.031)\end{array}$ & $\begin{array}{l}0.589^{*} \\
(0.034)\end{array}$ \\
\hline $\mathrm{N}$ & 596 & 597 & 598 & 588 \\
\hline \multicolumn{5}{|c|}{ Instructive paternalism only condition } \\
\hline Cameroon & $\begin{array}{c}0.017 \\
(0.055)\end{array}$ & $\begin{array}{c}0.052 \\
(0.045)\end{array}$ & $\begin{array}{c}0.068 \\
(0.060)\end{array}$ & $\begin{array}{l}-0.011 \\
(0.046)\end{array}$ \\
\hline Racial resentment & $\begin{array}{l}-0.603^{*} \\
(0.081)\end{array}$ & $\begin{array}{l}-0.506^{*} \\
(0.064)\end{array}$ & $\begin{array}{l}-0.661^{*} \\
(0.089)\end{array}$ & $\begin{array}{l}-0.396^{*} \\
(0.076)\end{array}$ \\
\hline $\begin{array}{l}\text { Cameroon X Racial } \\
\text { resentment }\end{array}$ & $\begin{array}{l}-0.032 \\
(0.104)\end{array}$ & $\begin{array}{l}-0.107 \\
(0.088)\end{array}$ & $\begin{array}{l}-0.112 \\
(0.116)\end{array}$ & $\begin{array}{l}-0.052 \\
(0.100)\end{array}$ \\
\hline Constant & $\begin{array}{l}0.625^{*} \\
(0.043)\end{array}$ & $\begin{array}{l}0.601^{*} \\
(0.032)\end{array}$ & $\begin{array}{l}0.724^{*} \\
(0.045)\end{array}$ & $\begin{array}{l}0.669^{*} \\
(0.034)\end{array}$ \\
\hline $\mathrm{N}$ & 547 & 546 & 549 & 535 \\
\hline \multicolumn{5}{|c|}{ Limiting paternalism condition } \\
\hline Cameroon & $\begin{array}{c}0.046 \\
(0.059) \\
\end{array}$ & $\begin{array}{c}0.084 \\
(0.057) \\
\end{array}$ & $\begin{array}{c}0.101 \\
(0.070)\end{array}$ & $\begin{array}{c}0.096 \\
(0.061)\end{array}$ \\
\hline Racial resentment & $\begin{array}{l}-0.518^{*} \\
(0.104)\end{array}$ & $\begin{array}{l}-0.506^{*} \\
(0.099)\end{array}$ & $\begin{array}{l}-0.637^{*} \\
(0.114)\end{array}$ & $\begin{array}{l}-0.329 * \\
(0.102)\end{array}$ \\
\hline $\begin{array}{l}\text { Cameroon X Racial } \\
\text { resentment }\end{array}$ & $\begin{array}{l}-0.009 \\
(0.119)\end{array}$ & $\begin{array}{l}-0.075 \\
(0.116)\end{array}$ & $\begin{array}{l}-0.120 \\
(0.141)\end{array}$ & $\begin{array}{l}-0.118 \\
(0.123)\end{array}$ \\
\hline Constant & $\begin{array}{l}0.551^{*} \\
(0.052)\end{array}$ & $\begin{array}{l}0.563^{*} \\
(0.049)\end{array}$ & $\begin{array}{l}0.697^{*} \\
(0.057)\end{array}$ & $\begin{array}{l}0.605^{*} \\
(0.051)\end{array}$ \\
\hline $\mathrm{N}$ & 554 & 552 & 555 & 550 \\
\hline
\end{tabular}

Note: Cells indicate coefficients and standard errors from linear regressions. All variables range from 0 to 1 . The analysis omitted the control condition, and the omitted category for the remaining conditions is the instructive paternalism only condition. Bold and asterisks indicate $\mathrm{p}<0.05$ with a two-tailed test. The sample was restricted to non-Hispanic whites. 
Table 8: Results for the black target county variable from Baker (2011) for high levels of racial resentment

\begin{tabular}{|c|c|c|c|c|}
\hline & $\begin{array}{l}\text { Support for } \\
\text { higher } \\
\text { foreign aid } \\
\text { spending in } \\
\text { general }\end{array}$ & $\begin{array}{l}\text { Support for } \\
\text { increasing } \\
\text { foreign aid } \\
\text { to the target } \\
\text { country }\end{array}$ & $\begin{array}{l}\text { Moral } \\
\text { obligation } \\
\text { to help } \\
\text { foreign poor } \\
\text { countries }\end{array}$ & $\begin{array}{c}\text { Foreign aid } \\
\text { to the target } \\
\text { country is } \\
\text { not wasted }\end{array}$ \\
\hline \multicolumn{5}{|c|}{ Full sample of whites } \\
\hline $\begin{array}{l}\text { Top } 50 \% \text { on the racial } \\
\text { resentment scale }\end{array}$ & $\begin{array}{c}0.020 \\
(0.019)\end{array}$ & $\begin{array}{c}0.021 \\
(0.019)\end{array}$ & $\begin{array}{c}0.035 \\
(0.022)\end{array}$ & $\begin{array}{l}-0.004 \\
(0.019)\end{array}$ \\
\hline $\begin{array}{l}\text { Top } 25 \% \text { on the racial } \\
\text { resentment scale }\end{array}$ & \multicolumn{4}{|c|}{ Same results as for the top $50 \%$} \\
\hline $\begin{array}{l}\text { Top } 10 \% \text { on the racial } \\
\text { resentment scale }\end{array}$ & $\begin{array}{c}0.026 \\
(0.035)\end{array}$ & $\begin{array}{c}0.037 \\
(0.034)\end{array}$ & $\begin{array}{c}0.013 \\
(0.044)\end{array}$ & $\begin{array}{l}-0.054 \\
(0.036)\end{array}$ \\
\hline $\begin{array}{l}\text { Top 5\% on the racial } \\
\text { resentment scale }\end{array}$ & \multicolumn{4}{|c|}{ Same results as for the top $10 \%$} \\
\hline \multicolumn{5}{|c|}{ White sample restricted to self-identified strong Democrats and not strong Democrats } \\
\hline $\begin{array}{l}\text { Top 50\% on the racial } \\
\text { resentment scale }\end{array}$ & $\begin{array}{c}0.020 \\
(0.038)\end{array}$ & $\begin{array}{c}0.029 \\
(0.035)\end{array}$ & \begin{tabular}{c|}
0.005 \\
$(0.043)$ \\
\end{tabular} & $\begin{array}{c}0.006 \\
(0.034)\end{array}$ \\
\hline $\begin{array}{l}\text { Top } 25 \% \text { on the racial } \\
\text { resentment scale }\end{array}$ & $\begin{array}{c}0.025 \\
(0.044)\end{array}$ & $\begin{array}{c}0.040 \\
(0.042)\end{array}$ & $\begin{array}{c}0.020 \\
(0.049)\end{array}$ & $\begin{array}{c}0.049 \\
(0.039)\end{array}$ \\
\hline $\begin{array}{l}\text { Top } 10 \% \text { on the racial } \\
\text { resentment scale }\end{array}$ & $\begin{array}{c}0.087 \\
(0.091)\end{array}$ & $\begin{array}{c}0.121 \\
(0.089)\end{array}$ & $\begin{array}{c}0.241 \\
(0.117)\end{array}$ & $\begin{array}{c}0.121 \\
(0.077)\end{array}$ \\
\hline $\begin{array}{l}\text { Top } 5 \% \text { on the racial } \\
\text { resentment scale }\end{array}$ & $\begin{array}{l}-0.078 \\
(0.043) \\
\end{array}$ & $\begin{array}{c}-0.078 \\
(0.043) \\
\end{array}$ & $\begin{array}{c}-0.145 \\
(0.066) \\
\end{array}$ & $\begin{array}{c}-0.163 \\
(0.087) \\
\end{array}$ \\
\hline \multicolumn{5}{|c|}{ White sample restricted to persons who self-identified as liberal or extremely liberal } \\
\hline $\begin{array}{l}\text { Top } 50 \% \text { on the racial } \\
\text { resentment scale }\end{array}$ & $\begin{array}{c}0.043 \\
(0.054)\end{array}$ & $\begin{array}{c}0.091 \\
(0.045)\end{array}$ & $\begin{array}{c}0.089 \\
(0.058) \\
\end{array}$ & $\begin{array}{c}0.054 \\
(0.043)\end{array}$ \\
\hline $\begin{array}{l}\text { Top } 25 \% \text { on the racial } \\
\text { resentment scale }\end{array}$ & $\begin{array}{c}0.068 \\
(0.076)\end{array}$ & $\begin{array}{c}0.102 \\
(0.062)\end{array}$ & $\begin{array}{c}0.050 \\
(0.087)\end{array}$ & $\begin{array}{c}0.066 \\
(0.067)\end{array}$ \\
\hline $\begin{array}{l}\text { Top } 10 \% \text { on the racial } \\
\text { resentment scale }\end{array}$ & $\begin{array}{c}0.130 \\
(0.102)\end{array}$ & $\begin{array}{c}0.130 \\
(0.099)\end{array}$ & $\begin{array}{c}0.304 \\
(0.159) \\
\end{array}$ & $\begin{array}{c}0.117 \\
(0.101)\end{array}$ \\
\hline $\begin{array}{l}\text { Top 5\% on the racial } \\
\text { resentment scale }\end{array}$ & \multicolumn{4}{|c|}{ Same results as for the top $10 \%$} \\
\hline
\end{tabular}

Note: Cells indicate coefficients and standard errors for the black country coefficient from linear regressions that included controls for experimental conditions. All variables range from 0 to 1 . Bold and asterisks indicate $p<0.05$ with a two-tailed test. The sample was restricted to non-Hispanic whites. Percentiles for subgroups are based on the distribution of racial resentment within that subgroup. 


\section{Supplemental Information for}

Does Racial Resentment Predict Anti-Black Discrimination?

I. Survey Information

\begin{tabular}{|l|r|r|c|c|}
\hline & \multicolumn{1}{|c|}{ Start Date } & End Date & $\begin{array}{c}\text { Completion } \\
\text { Rate }\end{array}$ & $\begin{array}{c}\text { Qualification } \\
\text { Rate }\end{array}$ \\
\hline Stephens 2011(b) [pretest] & July 1,2011 & July 9, 2011 & $54.5 \%$ & \\
\hline Stephens 2011(b) [posttest] & July 10, 2011 & July 17, 2011 & $72.9 \%$ & \\
\hline Mcllwain and Caliendo (2010) & March 23, 2010 & March 31, 2010 & $64.7 \%$ & $67.9 \%$ \\
\hline Baker (2011) & April 28, 2011 & May 9, 2011 & $67.0 \%$ & \\
\hline
\end{tabular}

II. Survey Items

Bold text indicates survey items that were used in the main analyses.

Stephens (2011b)

$\sim$ Pretest items for white respondents $\sim$

Here are a few statements about some things in our society. Please state whether you agree or disagree with each statement:

Q1a. Over the past few years, Blacks have gotten less than they deserve.

1. Strongly Agree

2. Agree

3. Don't Know

4. Disagree

5. Strongly Disagree

Q1b. Irish, Italian, Jewish, and many other minorities overcame prejudice and worked their way up. Blacks should do the same without any special favors.

1. Strongly Agree

2. Agree

3. Don't Know

4. Disagree

5. Strongly Disagree

Q1c. It's really a matter of some people not trying hard enough; if Blacks would only try harder they could be just as well off as Whites.

1. Strongly Agree

2. Agree

3. Don't Know

4. Disagree

5. Strongly Disagree 
Q1d. Generations of slavery and discrimination have created conditions that make it difficult for Blacks to work their way out of the lower class.
1. Strongly Agree
2. Agree
3. Don't Know
4. Disagree
5. Strongly Disagree

Q2. Imagine a seven-point scale on which the characteristics of the people in a group can be rated. A score of "1" means that you think almost all of the people in that group tend to be LAZY. A score of "7" means that you think most people in the group are HARDWORKING. A score of "4" means that you think most people in the group are not closer to one end or the other, and of course, you may choose any number in between. How well do the words "hardworking" and "lazy" describe Blacks as a group?

[Response options from ("lazy") to 7 ("hardworking")]

Q3. How important is being White to your ideas about politics?

1. Very important

2. Somewhat important

3. Not at all important

$\sim$ Posttest items for white respondents $\sim$

Q1. Please read over the list below, and choose as many (or as few) of the statements that you believe correctly describe the news article you just read?
1. It dealt with education
2. It showed a Black candidate
3. It dealt with the environment
4. It showed a candidate with glasses
5. It dealt with a candidate who tried to appeal to racial feelings

Q2. Please let us know if the story you read made you feel any of the following emotions? Anxious

Proud

Happy

Afraid

Uneasy

Disgusted

1. Yes

2. No

Q3. How likely is it that you would vote for Greg Davis for the U.S. House of Representatives?

1. Very Unlikely 

2. Unlikely
3. Don't Know
4. Likely
5. Very Likely

Q4. Do the following words describe candidate, Greg Davis extremely well, quite well, or not well at all:

Intelligent

Inexperienced

Trustworthy

Hardworking

Fair

\section{Extremely Well \\ 2. Quite Well \\ 3. Not Well At All}

Q5. Where would you place candidate Greg Davis on the following scale?
1. Extremely liberal
2. Liberal
3. Slightly liberal
4. Moderate, middle of the road
5. Slightly conservative
6. Conservative
7. Extremely conservative

Q6. In general, do you think the policies of Greg Davis will favor Whites over Blacks, Blacks over Whites, or will they treat both groups the same?

Will Favor Whites over Blacks

1. Treat Both Groups the Same

2. Will Favor Blacks over Whites

Q7a. On the average Blacks have worse jobs, income, and housing than White people. Do you think these differences are...

Because most Blacks don't have the chance for education that it takes to rise out of poverty?

1. Strongly Agree

2. Somewhat Agree

3. Don't Know

4. Somewhat Disagree

5. Strongly Disagree

Q7b. Because most Blacks just don't have the motivation or will power to pull themselves out of poverty?

1. Strongly Agree

2. Somewhat Agree 

3. Don't Know
4. Somewhat Disagree
5. Strongly Disagree

Q8a. On the average Black students have worse test scores and graduation rates than White students. Do you think these differences are....

Because of economic disadvantage?

1. Strongly Agree

2. Somewhat Agree

3. Don't Know

4. Somewhat Disagree

5. Strongly Disagree

Q8b. Because education is not a priority for Black parents?
1. Strongly Agree
2. Somewhat Agree
3. Don't Know
4. Somewhat Disagree
5. Strongly Disagree

Q8c. Because of biased/racist attitudes toward Blacks?

1. Strongly Agree

2. Somewhat Agree

3. Don't Know

4. Somewhat Disagree

5. Strongly Disagree

Q9. Should federal spending on education be increased, decreased, or kept about the same?
1. Increased
2. Kept About the Same
3. Decreased

Q10. On the average Black students have worse test scores and graduation rates than White students. This is called the "achievement gap." Should federal spending on the achievement gap be increased, decreased, or kept about the same?

1. Increased

2. Kept About the Same

3. Decreased

McIlwain and Caliendo (2010)

Q1. If you were able to vote in the election between David Jackson and Jim Herbert, for whom would you be most likely to vote based on what you know of the two candidates? 


\section{David Jackson \\ 2. Jim Herbert}

Q2. If the election were held today, how likely would you be to go to the polls to vote for whomever you indicated above?

1. Very likely

2. Somewhat likely

3. Not very likely at all

We'd like to get your feelings towards the candidates you saw in the campaign ads by rating of them using something we call the feeling thermometer. Ratings from 51 degrees to 100 degrees mean that you feel favorable and warm toward the candidate. Ratings from 0 degrees to 49 degrees mean that you don't feel favorable toward the candidate. You would rate the candidate at the 50 degree mark if you don't feel particularly warm or cold toward him.

Q3a. David Jackson

Q3b. Jim Herbert

Q4. Based on what you know about the two candidates, how closely does each of them match your idea of what your congressman should be like?

[Item asked about David Jackson and asked separately about Jim Herbert]

1. Extremely closely

2. Very closely

3. Somewhat closely

4. Not very closely at all

Q5. In the advertisement you viewed from David Jackson, would you say that he was making a racial appeal?

1. Definitely

2. Maybe

3. Definitely not

4. Don't know

Q6. In the advertisement you viewed, from Jim Herbert, would you say that he was making a racial appeal?

1. Definitely

2. Maybe

3. Definitely not

4. Don't know

5.

We would like to ask you some questions about your feelings and beliefs about black Americans and members of other racial groups. 
Q7. It's really a matter of some people not trying hard enough; if blacks would only try harder they could be just as well off as whites.
1. Strongly agree
2. Somewhat agree
3. Somewhat disagree
4. Strongly disagree

Q8. Irish, Italian, Jewish and many other minorities overcame prejudice and worked their way up. Blacks should do the same.
1. Strongly agree
2. Somewhat agree
3. Somewhat disagree
4. Strongly disagree

Q9. Some say that black leaders have been trying to push too fast. Others feel that they haven't pushed far enough. What do you think?

1. Trying to push too fast

2. Going too slowly

3. Moving at about the right speed

Q10. How much of the racial tension that exists in the United States today do you think blacks are responsible for creating?

1. All of it

2. Most of it

3. Some of it

4. Not much of it at all

Q11. How much discrimination against blacks do you feel there is in the United States today, limiting their chances to get ahead?

1. A lot

2. Some

3. Just a little

4. None at all

Q12. Generations of slavery and discrimination have created conditions that make it difficult for blacks to work their way out of the lower class.
1. Strongly agree
2. Somewhat agree
3. Somewhat disagree
4. Strongly disagree

Q13. Over the past few years, blacks have gotten less than they deserve.

1. Strongly agree

2. Somewhat agree

3. Somewhat disagree

4. Strongly disagree 
Q14. Over the past few years, blacks have gotten more economically than they deserve.

1. Strongly agree

2. Somewhat agree

3. Somewhat disagree

4. Strongly disagree

Q15. Some people seem to follow what's going on in government and public affairs most of the time, whether there's an election going on or not. Others aren't that interested. Would you say you follow what's going on in government and public affairs most of the time, some of the time, only now and then, or hardly at all?

1. Most of the time

2. Some of the time

3. Only now and then

4. Hardly at all

\section{Baker (2011)}

Q1. Overall, each year the US government gives about \$40 of each American's income to foreign countries. Many people think this is too low, others think it is too high, and still others think it is about right. How much per American do you think our government should spend on foreign aid?

1. \$0. The U.S. should not give any foreign aid.

2. $\$ 1$ to $\$ 19$. The U.S. should lower the amount by a lot.

3. $\$ 20$ to $\$ 39$. The U.S. should lower the amount by a little.

4. \$40. The current amount is about right.

5. $\$ 41$ to $\$ 59$. The U.S. should raise the amount by a little.

6. $\$ 60$ to $\$ 79$. The U.S. should raise the amount by a lot.

7. $\$ 80$ or more. The U.S. should raise the amount by a huge sum.

Q2. Now we'd like to ask your opinion about foreign aid in a slightly different way. What about aid to [Cameroon/Moldova/a poor country] where the average person survives on the US equivalent of about $\$ 1,800$ per year, about $\$ 50$ of which comes from wealthy foreign countries as aid. Do you think that U.S. spending on foreign aid to [Cameroon/Moldova/poor countries] should increase, decrease or be kept about the same? If you think it should increase or decrease, please specify by how much.

1. It should decrease to zero.

2. It should decrease a lot.

3. It should decrease a little.

4. It should stay the same.

5. It should increase a little.

6. It should increase a lot.

7. It should increase by a huge sum. 
Keeping [in mind the poor countries of Africa/in mind the poor countries of Eastern Europe/poor countries in mind], read each of the following statements and then decide how much you agree or disagree with each one:

Q3. The only way poor countries could grow richer is with financial help from rich countries.

Q4. Because of difficult economic circumstances, people in poor countries are unable to help themselves get richer.

Q5. There is little that people in poor countries can do by themselves to improve their livelihoods.

[Note: The questionnaire and dataset had no Q6 item.]

Q7. It's really a matter of some people not trying hard enough. If people in poor countries would only try harder, they could be just as well off as the United States.

Q8. Generations of colonialism and economic exploitation by rich countries have kept poor countries from becoming richer.

Q9. The US has a moral obligation to help foreign poor countries.

[Options for items Q3 to Q9 were: Agree strongly, Agree somewhat, Neither agree nor disagree, Disagree somewhat, and Disagree strongly]

Q10. There are also different opinions about how well foreign aid is used by the poor people and countries it is supposed to benefit. Do you think that foreign aid is used [in Cameroon/in Moldova] to genuinely improve the lives of poor people or is it wasted? Please indicate below the amount of all foreign aid that you think is used well [in Cameroon/in Moldova].

[Options for item Q10 were: All of it is used well, none of it is wasted; Most of it is used well, a little of it is wasted; Half is used well, half is wasted; A little of it is used well, most is wasted; None of it is used well, all of it is wasted]

Q11. Finally, we'd like to get your view on what quality of life is like in poor countries. In [Cameroon,/Moldova,/a poor country] where the average person survives on the US equivalent of $\$ 1,800$ per year ( $\$ 5$ per day), about what percent of households have the following?

At least two meals per day. 

1. $0 \%$
2. $10 \%$
3. $20 \%$
4. $30 \%$
5. $40 \%$
6. $50 \%$
7. $60 \%$
8. $70 \%$
9. $80 \%$
$10.90 \%$
11. $100 \%$

Q12. In [Cameroon,/Moldova,/a poor country] where the average person survives on the US equivalent of $\$ 1,800$ per year ( $\$ 5$ per day), about what percent of households have the following?

Indoor plumbing (i.e., flushing toilets and working water faucets).

1. $0 \%$

2. $10 \%$

3. $20 \%$

4. $30 \%$.

5. $40 \%$

6. $50 \%$

7. $60 \%$

8. $70 \%$

9. $80 \%$

10. $90 \%$

11. $100 \%$ 